\title{
Predictors of skilled assistance seeking behavior to pregnancy complications among women at southwest Ethiopia: a cross-sectional community based study
}

Serawit Lakew ${ }^{1 *}$, Erdaw Tachbele ${ }^{2}$ and Terefe Gelibo ${ }^{3}$

\begin{abstract}
Background: In Ethiopia, about 20,000 women die each year from complications of pregnancy and child birth with many more maternal morbidities occurring for each maternal deaths. This makes Ethiopia one of the highest countries for maternal deaths in the developing world. This study attempted to assess women's skilled assistance seeking behaviour for pregnancy complications among those who gave birth.
\end{abstract}

Method: A cross-sectional community based study was conducted among women who gave birth within one year regardless of their delivery place. The study was carried out in fifteen randomly selected villages at Arba Minch Zuria district, south west Ethiopia. Data was collected house-to-house using a pretested Amharic questionnaire. During the survey, 798 women were interviewed. Logistic regression model was applied to control confounders.

Results: Out of the total sample, $344(43.1 \%)$ respondents reported at least any one of the pregnancy complications faced in the recent pregnancy. The most common complications reported were malaria (57\%), nausea/vomiting (47.1\%) and severe head ache (29.1\%). of those women who faced complications, around 254 (73.8 \%) sought assistance from a skilled provider. Ninety (26.2 \%) of the respondents sought assistance either from unskilled provider or home based self-care. Unable to understand the seriousness of the complications, thought as unnecessary, and family disapproval were the major reasons for not seeking care from skilled providers. Belonging to monthly household income \$US25- $100(\mathrm{AOR}=3.4,95 \% \mathrm{Cl} ; 1.04,11.4)$, getting antenatal care from a skilled provider ( $A O R=10.6,95 \% \mathrm{Cl}$; 3.3, 34.5), Women in the age 20-34 years old (AOR $=3.8 ; 95 \% \mathrm{Cl}, 1.2,12.3)$, Availability of transport access (AOR $=72.2 ; 95 \% \mathrm{Cl} ; 17.2,303.5)$ were significantly associated with seeking assistance from a skilled provider.

Conclusions: Nearly half (43.1\%) of the women had faced pregnancy complications to the recent birth of last one year. Majority $\left(2 / 3^{\text {rd }}\right)$ of the women who reported complications sought skilled assistance. Family, income, transport issue and antenatal care use were independent predictors for skilled assistance from skilled provider.

Keywords: Predictors, Skilled assistance, Pregnancy complications, Women, South west Ethiopia

\footnotetext{
*Correspondence: Iserawit@yahoo.com

${ }^{1}$ Department of Nursing and Midwifery, Arba Minch College of Health

Sciences, Arba Minch, South West Ethiopia

Full list of author information is available at the end of the article
} 


\section{Background}

An estimated 287,000 maternal deaths occurred in 2010 worldwide, which is $50 \%$ reduction from 1990 baseline [1]. In Ethiopia, about 20,000 women die each year from complications of pregnancy and child birth with many more maternal morbidities occurring for each maternal death. This makes Ethiopia to be one of the countries with the highest maternal mortality ratio, which is 676 per 100,000 live births, EDHS 2011 Report [2].

All pregnant women are at risk of developing lifethreatening complications and around $15 \%$ of these women develop complications [3]. These complications can involve the mother's health, the baby's, or both. Pregnancy complications can range from mild and annoying discomforts to severe, sometimes life-threatening, illnesses [4]. Some of these life threatening complications range from vaginal bleeding up to high fever. And non- life threatening complications range from nausea and/or vomiting to varicus vein [4-8].

The outcomes of most pregnancy complications are unpredictable unless they are managed by appropriate health care providers. Studies indicated that life threatening maternal complications may end up in the death of the mother within two hours of onset in postpartum hemorrhage to six days in puerperal sepsis [9]. Previous studies have identified factors predicting the care seeking behaviors for pregnancy complications include higher educational status, ANC utilization, near distance to health facility, availability of transport system, small family size, previous pregnancy experience, and good economic status have been observed with high probability of skilled assistance seeking for pregnancy complications $[2,5,6,9-16]$. According to the latest Ethiopian demographic and health survey report, skilled maternal health services utilization is still low. So, this study was aimed to determine skilled assistance seeking behaviour for pregnancy complications that women faced (for any one or more complications) among those who were permanent dwellers of Arba Minch Zuria district, Gamo Gofa Zone.

\section{Methods}

\section{Study setting and design}

A community based cross-sectional study was conducted from April 15 to Jun 5, 2014 in Arba Minch Zuria district, Gamo Gofa Zone, Southwest Ethiopia, which is found in the Great Rift Valley, located $505 \mathrm{kms}$ south of Addis Ababa [17]. In the district there were 30 kebeles and a total population of 195,315 , of whom 95,704 were men and 99,611 women [18]. The district had 38,663 total estimated households and 9,766 estimated population of women with under one year child or still birth in the last one year prior to this survey $(2.4 \%$ for $<1$ year and $2.6 \%$ for still births, in the total population). The health infrastructure of the district includes six health centers, thirty health posts, one Hospital of the governmental, and fifteen medium level and lower level private clinics [17-19].

\section{Sample size and sampling procedure}

The sample size was determined taking $52.1 \%$ estimated prevalence of skilled assistance at Northwest Ethiopia (since northwest had nearly similar health infrastructure, socio-economy and culture with southwest) [9]. Assuming $5 \%$ margin of error, $95 \%$ confidence level, $5 \%$ nonresponse rate, and design effect, the total sample size was 804. For sampling, multistage cluster sampling technique was used. Out of 30 clusters of kebeles (kebele is the lowest administrative unit in Ethiopia) in the district, 15 kebeles were randomly selected for this study (that is $50 \%$ of the cluster, clusters were assumed homogenous to the study variables). Within each of these 15 kebeles, 3 villages (Villages are sub-divisions of one kebele) were selected. This is, again, $50 \%$ selection out of an average six locations (villages). Sampling frame of household was obtained for each kebeles from district administration Health Office. For selection of respondents in each kebeles and villages, Population proportionate to size (PPS) technique was employed. The respondents from the sampling frame of the eligible households were selected by lottery method. One center of the village was selected in each village. From this center spinning a pen technique was used for selection of walking direction by data collectors. The household in the direction of Tip of pen was started with a nearby first. After a successful interview of each household, the interviewer continued to the immediate nearby household of the eligible and selected woman until the required sample size was achieved in each of the kebele.

\section{Measurement}

Data were collected using interviewer administered questionnaires. The data collection tool was adapted from DHS and other literatures on maternal health surveys [6]. The main foci of the questionnaire were on sociodemographic characteristics; knowledge and experiences related to pregnancy complications; and use of skilled maternal care services. Some of questions presented include: the type of facility visited, type of care provider; decision maker to seek care; transport access; and distance from home to health facility. A maximum effort was made to ensure privacy during interview. Data collectors and supervisors were hired from nursing background. They have given five days training on data collection techniques and objectives of the study. The interviews were made in Amharic local language. Translations and re-translations were performed by experts of both English and Amharic language. 


\section{Statistical analysis}

The data were coded, edited and entered into Epi-info version 3.5.2, cleaned and analyzed by SPSS for windows version 20. Frequencies, proportions and summary statistics were used to describe the study population in relationship to the relevant variables. Statistical analysis had three steps: first association was done between potential predictors of socio-demography and Skilled Antenatal visit (by Doctor, Nurse, or Midwife) for skilled assistance seeking to pregnancy complications using bivariate analysis and $95 \%$ confidence intervals to show existence of bivariate association. Next, to identify the independent contribution of each variable multivariate logistic regression model was used for the variables having association $(p<0.05)$ in bivariate logistic regression model. Finally, it was evaluated that variables identified as associated $(P<0.05)$ with the outcome variable in the multivariate analysis were used to predict the existence of association.

\section{Data quality control}

Data Collection tool was adjusted for locally suitability and pretested. Every day completed questionnaires were reviewed and checked for completeness and relevance by the supervisors and Principal investigators. All the necessary feedback was offered to data collectors in the next morning before the actual procedure. Data checked for completeness, coded, entered into computer, cleaned and frequency checked for outliers and missing values before analysis.

\section{Ethical issues}

Ethical clearance was obtained from Addis Ababa University, School of Allied Health Sciences Institutional Review Board (IRB). The study was commenced after letter of cooperation written to kebele Health Extension Workers (HEW) from District Health Office. Informed verbal consent was secured for each study subjects. Each respondent was informed about the objective of the study and assurance of confidentiality, risks and benefits.

\section{Definitions}

Income $=$ measured based on respondents estimated household monthly income.

Transport access = includes availability of roads and vehicle transport (bajaj, ambulance, car, or bus), but mule transport excluded.

Knowledge of complications = respondents who responded the median and more of the complications from the list for knowledge were designated as knowledgeable and those below median were not knowledgeable.

Sought skilled assistance $=$ respondents who sought assistance from Doctors, Nurses, Midwifes, or Health Officers for any one of the recent pregnancy complications.

\section{Results \\ Socio-demographic characteristics of the study participants}

A total of 798 women participated in the survey making the response rate of $99 \%$. Majority $(78.2 \%)$ of the women at the time of last pregnancy were 20-34 years of age with mean age of 25.7 years \pm 6.3 standard deviation and range of 14-49 years of age. The dominant ethnicity in the surveyed area was Gamo (73.4 \%), followed by Ganta (13\%) and Welayta (11.5\%) ethnic groups respectively. Majority $(99.7 \%)$ of the respondents were a Christian religion follower that belongs to either Christian orthodox (28.3\%) or Christian protestant $(71.4 \%)$. The median monthly income of the family was \$US45 (Table 1).

\section{Complications women faced}

Out of 798 respondents included in the study, $43.1 \%$ (344 out of 798 women) had encountered at least any one of the problems either life threatening or non-life threatening or both, last during the recent pregnancy in the last 12 months. Of this complications, about 334 $(97.1 \%)$ reported life threatening pregnancy complications and 180 (52.3\%) non-life threatening pregnancy complications (since most respondents had mixed complications percentage was greater than 100 and number greater than 344). Mean $( \pm S D)$ occurrence $2 \pm 1$ for life threatening pregnancy complications and mean $( \pm \mathrm{SD})$ occurrence $0.72 \pm 0.8$ for non-life threatening pregnancy complications. The major life threatening complications faced at the last pregnancy were malaria 196 (57 \%); severe headache 100 (29.1\%); and severe lower abdominal Pain 86 (25\%) (Fig. 1). Nausea and vomiting occurred on $162(47.1 \%)$ of mothers, which was the larger nonlife threatening pregnancy complication (Fig. 2).

\section{Women assistance seeking behaviour}

Out of women faced complications, $73.8 \%$ (254 out of 344) sought skilled assistance, $16.9 \%$ (58 out of 344 women) sought unskilled assistance (sought assistance from unskilled provider, such as HEW, TBA, and other unqualified health worker), and the remaining $9.3 \%$ (32 out of 344 women) did seek no assistance at all or had home based self-care (Table 2).

As summarized in Table 3, of the total 254 respondents who had skilled assistance to pregnancy complications, about 170 (72.3\%) respondents had ANC by a skilled provider in the recent pregnancy. Out of 58 respondents who had unskilled assistance to pregnancy complications, about $52(89.7 \%)$ respondents had unskilled ANC follow up in the recent pregnancy.

\section{Predictors of skilled assistance seeking behaviour}

The multivariable analysis carried out using binary logistic regression indicated that four variables: Family 
Table 1 Frequency and percentage distribution of respondents according to selected socio-demographic characteristics, Arba Minch Zuria district, south west Ethiopia, 2014

\begin{tabular}{|c|c|c|c|}
\hline Characteristics & & $\begin{array}{l}\text { Frequency (n), } \\
n=798\end{array}$ & Percentage (\%) \\
\hline \multirow[t]{4}{*}{ Age (years) } & $15-19$ & 96 & 12.0 \\
\hline & $20-34$ & 624 & 78.2 \\
\hline & $35-49$ & 78 & 9.8 \\
\hline & Mean \pm std. dev $^{a}$ & $25.7 \pm(6.3)$ & \\
\hline \multirow[t]{3}{*}{ Religion } & Protestant & 570 & 71.4 \\
\hline & Orthodox & 226 & 28.3 \\
\hline & Others $^{b}$ & 2 & 0.3 \\
\hline \multirow[t]{4}{*}{ Ethnicity } & Gamo & 586 & 73.4 \\
\hline & Ganta & 104 & 13.0 \\
\hline & Welayta & 92 & 11.5 \\
\hline & Others $^{c}$ & 16 & 2.0 \\
\hline \multirow[t]{2}{*}{ Marital Status } & Married & 770 & 96.5 \\
\hline & Others $^{d}$ & 28 & 3.5 \\
\hline \multirow{4}{*}{$\begin{array}{l}\text { Family Monthly } \\
\text { Income (\$US) }\end{array}$} & $<25$ & 300 & 37.6 \\
\hline & $25-100$ & 394 & 49.4 \\
\hline & $>100$ & 104 & 13 \\
\hline & Median & 45 & \\
\hline \multirow[t]{4}{*}{ Women Education } & No Education & 192 & 24.1 \\
\hline & Primary & 442 & 55.4 \\
\hline & Secondary & 124 & 15.5 \\
\hline & College/University & 40 & 5 \\
\hline \multirow[t]{5}{*}{ Birth Order } & 1 & 230 & 28.8 \\
\hline & $2-3$ & 394 & 49.4 \\
\hline & $4-5$ & 126 & 15.8 \\
\hline & $6+$ & 48 & 6 \\
\hline & Median & 2 & \\
\hline \multirow{3}{*}{$\begin{array}{l}\text { No transport } \\
\text { access problem }\end{array}$} & Yes & 202 & 62.7 \\
\hline & No & 120 & 37.3 \\
\hline & Missing $^{f}$ & 476 & 59.6 \\
\hline
\end{tabular}

N.B: ${ }^{a}$ standard deviation, ${ }^{b}$ muslim, ${ }^{\mathrm{C}}$ Zayse, Oromo, Amhara and Ganjule, ${ }^{d}$ widowed, divorced, single, ${ }^{,}$transport access is for motor vehicle transport, existence of road to the facility, (foot and mule transport excluded),

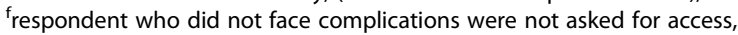
transport access is difficult to say yes or no(not adequate, but sometimes used) were also under missed

monthly income, having ANC by a skilled provider, age of respondents, and transportation access to the facility were the factors found to be significantly associated with seeking assistance from a skilled provider. Women who had antenatal care by a skilled provider found to be 10.6 times $(\mathrm{AOR}=10.6,95 \% \mathrm{CI} ; 3.3,34.5)$ more likely to seek assistance from a skilled provider for pregnancy complications as compared to women who did not have antenatal care by a skilled provider. Women with household monthly income of $\$$ US25-100/month were about 3.4 times (AOR $=3.4,95 \% \mathrm{CI} ; 1.04,11.4)$ more likely to be assisted by a skilled provider at the time of pregnancy complications than women who had monthly household income below $\$$ US25. Women who were in the age 2034 years were about 3.8 times $(\mathrm{AOR}=3.8 ; 95 \% \mathrm{CI}, 1.2$, 12.3) more likely to be assisted by a skilled provider at the time of pregnancy complications as compared to older women (below 20 years of age) with complications. Similarly, women who had access to transportation to go to the facility were 72.2 times $(\mathrm{AOR}=72.2 ; 95 \% \mathrm{CI}$, 17.2, 303.5) more likely to be assisted by a skilled provider for pregnancy complications as compared to those women of the counterparts (Table 4).

\section{Discussion}

This study showed, $43.1 \%$ of the respondents had encountered any one or more of the pregnancy complications. This finding was lower from an Indian slum study (43.1 \% VS 60 \%) [7], but higher from that of North Ethiopian study which was $28.5 \%$ [9]. This increased perception for reporting may be related to increased health extension workers and the recent network of health development army in the community and the increase in health services infrastructures in Ethiopia. Lower finding comparative to Indians may show low reporting of the complications \& its low perceived severity as related to Indian pregnant women's, though had increasing perception and reporting seen from time to time in Ethiopia.

In this study, skilled ANC attendance and skilled assistance seeking to pregnancy complications was significantly associated (AOR $=10.6,95 \% \mathrm{CI}$; 3.3, 34.5). This finding was consistent with other studies in that the contribution of ANC by skilled provider to further maternal service utilizations was observed in previous studies in Ethiopia [9, 20] and outside Ethiopia [13, 21]. This may be due to effective counseling services given to pregnant women during ANC visits by a skilled provider better motivated the women for further assistance as compared to unskilled ANC provider (HEW and TBA). There is no doubt that this is because skilled providers were more skillful than unskilled providers for effective and more quality counseling on pregnancy complications and its symptoms.

This study revealed that some of the women who reported complications did not seek services due to reasons, like did not see seriousness of the problems, seen as not necessary, and because family did not allow to seek assistance. These finding agreed with the findings outside Ethiopia [7, 12, 22], but differed with the previous findings of home study [9] and outside [11] in that inability to judge the graveness of condition, distance/ transport problems, lack of money/cost considerations, 


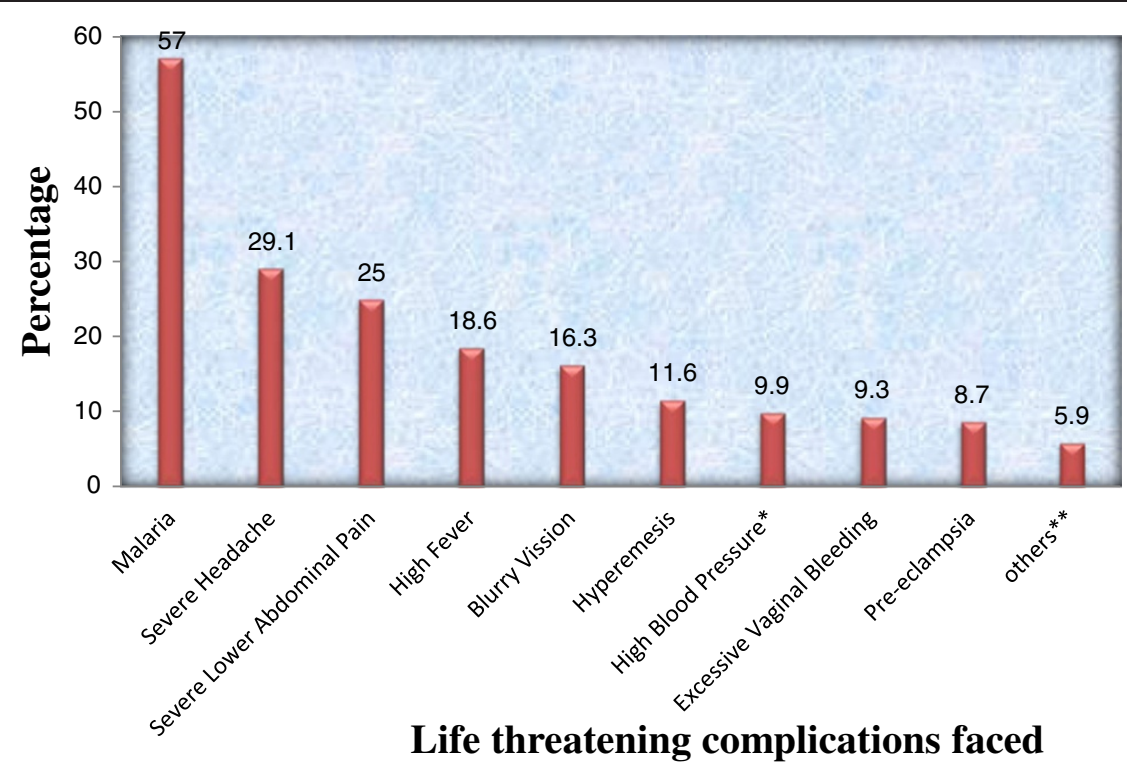

N.B *High Blood Pressure without pre-eclampsia, **Foul smelling vaginal discharge, Convulsion/Eclampsia, and malposition after 36wks of gestation

Fig. 1 Percent distribution of life threatening pregnancy complications reported in the recent pregnancy, Arba Minch Zuria District, South West Ethiopia, 2014

and use of traditional options at home, inadequate training of health personnel and lack of health insurance were observed. These differences may be due to cultural differences of inhabitants from region to region with in the home country and some cultural similarities of home country with outside home to influence on not to seek assistance to pregnancy complications.

In current study, it was identified that there was statistically significant association between medium household monthly income and skilled assistance to pregnancy complications (AOR $=3.4,95 \% \mathrm{CI} ; 1.04,11.4)$. This was inline with previous findings at home [9], while not in agreement with the findings abroad $[6,7,15]$ in that skilled assistance was higher among wealthier households. The difference may be due to difference in income classifications of the studies. Household monthly income of this study was classified as below \$US 25, \$US 25-100 and above \$US 100.

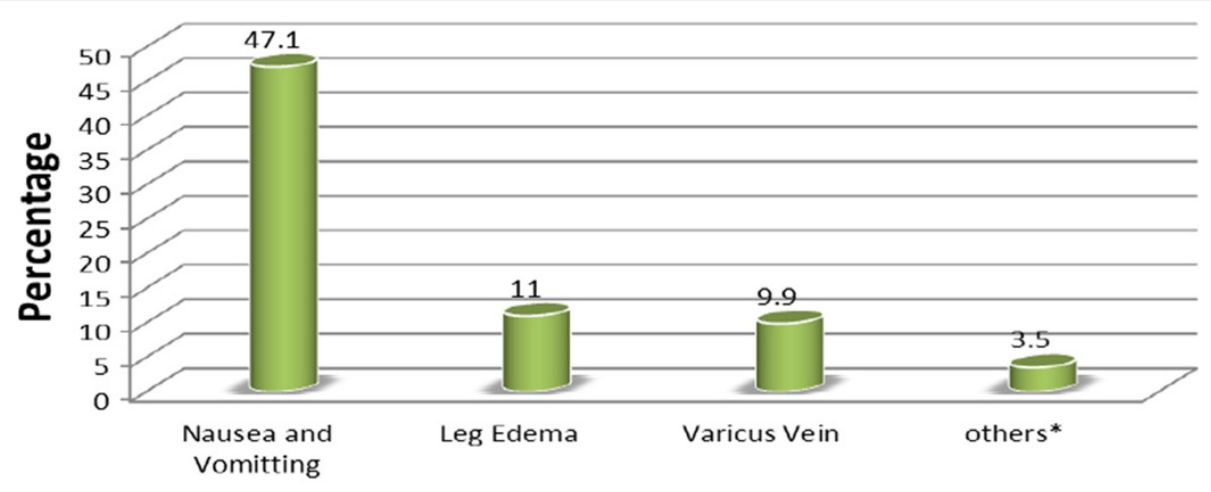

Non-lifethreatening complications faced

N.B: * gastric pain, weakness, mild depression and diarrhea

Fig. 2 Percentage distribution of non-life threatening pregnancy complications reported in the recent pregnancy, Arba Minch Zuria District, South West Ethiopia, 2014 
Table 2 Percent distribution of the respondents by seeking health care assistance to the complication faced last in the recent pregnancy, Arba Minch Zuria district, south west Ethiopia, 2014

\begin{tabular}{|c|c|c|c|c|c|}
\hline \multirow[t]{2}{*}{ Characteristics } & & \multicolumn{4}{|c|}{ Seeking health care assistance } \\
\hline & & $\begin{array}{l}\text { Skilled assistance }{ }^{a} \\
n=254(73.8 \%)\end{array}$ & $\begin{array}{l}\text { Unskilled assistance }^{\mathrm{b}} \\
n=58(16.9 \%)\end{array}$ & $\begin{array}{l}\text { No-assistance }^{c} \text { (self-care) } \\
N=32(9.3 \%)\end{array}$ & $\begin{array}{l}\text { Total } \\
n=344(100 \%)\end{array}$ \\
\hline \multirow[t]{3}{*}{ Age (years) } & $15-19$ & $30(8.7)$ & $4(1.2)$ & $2(0.6)$ & $36(10.5)$ \\
\hline & $20-34$ & $206(59.9)$ & $42(12.2)$ & $24(7)$ & $272(79.1)$ \\
\hline & $35-49$ & $18(5.2)$ & $12(3.5)$ & $6(1.7)$ & $36(10.4)$ \\
\hline \multirow[t]{4}{*}{ Birth order } & 1 & $62(18)$ & $12(3.5)$ & $4(1.2)$ & $78(22.7)$ \\
\hline & $2-3$ & $122(35.5)$ & $26(7.5)$ & $18(5.2)$ & $166(48.2)$ \\
\hline & $4-5$ & $52(15.1)$ & $16(4.7)$ & $2(0.6)$ & $70(20.4)$ \\
\hline & $6+$ & $18(5.2)$ & $4(1.2)$ & $8(2.3)$ & $30(8.7)$ \\
\hline \multirow[t]{3}{*}{ Family monthly income (\$US) } & $<25$ & $84(24.4)$ & $26(7.6)$ & $18(5.2)$ & $128(37.2)$ \\
\hline & $25-100$ & $146(42.4)$ & $18(5.2)$ & $12(3.5)$ & $176(51.1)$ \\
\hline & $>100$ & $24(7)$ & $14(4.1)$ & $2(0.6)$ & $40(11.7)$ \\
\hline \multirow[t]{4}{*}{ Women education } & No education & 66 (19.2) & $12(3.5)$ & $8(2.3)$ & $86(25.0)$ \\
\hline & Primary & $134(38.9)$ & $44(12.8)$ & $16(4.7)$ & $194(56.4)$ \\
\hline & Secondary & $34(9.9)$ & $2(.6)$ & $8(2.3)$ & $44(12.8)$ \\
\hline & College/university & $20(5.8)$ & $0(00.0)$ & $0(0.0)$ & $20(5.8)$ \\
\hline \multirow[t]{2}{*}{ Knowledge complications } & Yes & $188(54.6)$ & $28(8.2)$ & $22(6.4)$ & $238(69.2)$ \\
\hline & No & $66(19.2)$ & $30(8.7)$ & $10(2.9)$ & $106(30.8)$ \\
\hline
\end{tabular}

N.B: ${ }^{a}$ skilled assistance may include unskilled, ${ }^{b}$ assisted by HEWs, TBAs and other unskilled Health worker, chad home based self -care, left unassisted at all, home assisted by husband

Women in the age group 20-34 years old had statistically significant association with seeking skilled assistance to pregnancy complications $(\mathrm{AOR}=3.8 ; 95 \% \mathrm{CI}$, $1.2,12.3)$. This was not agreeing Indian study finding [7] in that more use of skilled assistance observed with greater maternal age, such as more than 35 years. The difference could be due to high number of women in the last one year post birth exist in the age group of 20-34 years in this study district as compared to Indians

Table 3 Percent distribution of skilled and unskilled assistance to pregnancy complications by four choices of ANC provider in the recent pregnancy, Arba Minch Zuria district, south west Ethiopia, 2014

\begin{tabular}{llll}
\hline ANC provider & \multicolumn{3}{l}{ Sought assistance } \\
\cline { 2 - 4 } & Category & $\begin{array}{l}\text { Skilled assistance } \\
n(\%)\end{array}$ & $\begin{array}{l}\text { Unskilled assistance }^{b} \\
n(\%)\end{array}$ \\
\hline Skilled ANC & Yes & $166(65.4 \%)$ & $4(6.9 \%)$ \\
& No & $88(34.6 \%)$ & $54(93.1 \%)$ \\
\multirow{3}{*}{ Unskilled ANC } & Total & $254(100 \%)$ & $58(100 \%)$ \\
& Yes & $84(33.1 \%)$ & $52(89.7 \%)$ \\
& No & $170(66.9 \%)$ & $6(10.3 \%)$ \\
& Total & $254(100 \%)$ & $58(100 \%)$
\end{tabular}

N.B: ${ }^{a}$ skilled assistance $=$ had sought health care assistance by doctor, nurse, midwife, or Health Officer

bUnskilled assistance $=$ had sought health care assistance by HEWs, TBAs, or other unqualified provider outside finding. Moreover, the increased primary education in Ethiopia might have contributed for the increased seeking behavior.

Women who had transportation access to the health facility had statistically significant association $(\mathrm{AOR}=$ 72.2; $95 \% \mathrm{CI}, 17.2,303.5$ ) with skilled assistance by a skilled provider. This is in-line with the findings in Upper(southern) Rural Egypt study [16] in that transportation service accessibility problems were seen as barrier to seeking skilled assistance to pregnancy complications in both studies.

\section{Conclusion and recommendations}

Women who reported at least any one of the problems faced in the recent pregnancy were highest as compared to previous prevalence. The most common complications reported were Malaria, Nausea/Vomiting and severe head ache. Of women faced complications, more than seven out of ten sought assistance from a skilled provider. Belonging to income group $\$$ US25 to 100 , getting antenatal care from a skilled provider, maternal age of 20-34 years, transport access were independent predictors of seeking assistance from a skilled provider. Community awareness program should focus on life threatening pregnancy complications on seeking assistance. 
Table 4 Adjusted and unadjusted odds ratio of logistic regression model showing effects of predictor variables on the likely hood of skilled assistance for pregnancy complications, Arba Minch Zuria district, south west Ethiopia, 2014

\begin{tabular}{|c|c|c|c|c|c|c|c|c|c|}
\hline \multirow[t]{3}{*}{ Variables } & & \multicolumn{8}{|c|}{ Assisted by skilled provider } \\
\hline & & \multirow{2}{*}{$\begin{array}{l}\text { Yes } \\
n=254\end{array}$} & \multirow{2}{*}{$\begin{array}{l}\text { No } \\
n^{\mathrm{b}}=90\end{array}$} & \multirow[t]{2}{*}{ COR } & \multicolumn{2}{|c|}{$95 \% \mathrm{Cl}$} & \multirow[t]{2}{*}{$\mathrm{AOR}$} & \multicolumn{2}{|c|}{$95 \% \mathrm{Cl}$} \\
\hline & & & & & Lower & Upper & & Lower & Upper \\
\hline \multirow[t]{3}{*}{ Age (years) } & $15-19$ & 30 & 8 & 5.8 & 0.99 & 34.4 & 4.2 & 0.6 & 28.1 \\
\hline & $20-34$ & 206 & 64 & $3.6^{*}$ & 1.2 & 10.8 & $3.8^{*}$ & 1.2 & 12.3 \\
\hline & $35-49$ & 18 & 18 & $1.0+$ & & & $1.0+$ & & \\
\hline \multirow[t]{3}{*}{ Family Monthly Income (\$US) } & $<25$ & 84 & 42 & $1.0+$ & & & $1.0+$ & & \\
\hline & $25-100$ & 146 & 32 & $5.4^{*}$ & 0.6 & 16.8 & $3.4^{*}$ & 1.01 & 11.4 \\
\hline & $>100$ & 24 & 16 & 2.0 & 0.68 & 5.89 & 5.7 & .59 & 55.5 \\
\hline \multirow[t]{2}{*}{ Had ANC by Skilled Provider } & Yes & 166 & 22 & $12.7^{*}$ & 4.1 & 38.7 & $10.6^{*}$ & 3.3 & 34.5 \\
\hline & No & 88 & 68 & $1.0+$ & & & $1.0+$ & & \\
\hline \multirow[t]{2}{*}{ Knowledge of Complications } & Yes & 188 & 62 & $0.23^{\mathrm{a}}$ & 0.1 & 0.52 & 1.13 & 0.2 & 6.4 \\
\hline & No & 66 & 28 & $1.0+$ & & & $1.0+$ & & \\
\hline \multirow[t]{2}{*}{ No transport access problem } & Yes & 198 & 6 & $33^{*}$ & 9.3 & 116.6 & $72.2^{*}$ & 17.2 & $303.5^{c}$ \\
\hline & No & 56 & 84 & $1.0+$ & & & $1.0+$ & & \\
\hline
\end{tabular}

N.B: * Statistically Significant Association $(p<0.05)$

${ }^{\text {a }}$ Significant by binary analysis

${ }^{b}$ includes unskilled assistance, or self care or no assistance at all

clarge upper limit

+ Reference category

\section{Strengths of the study}

Being community based is an advantage for representing the community of woreda as compared to being facility based. Moreover, Professional data collectors (being midwifery nurse) used was an advantage for effective collection of medical information from the respondent's.

\section{Limitations of the study}

The cause effect relationship for all significant associations may have a chicken egg dilemma, since this is cross-sectional study. The reliability of self-reported complications based on a woman's recall may be limited compared to the results of medical examinations at health care facilities. Judging the severity of the illness is also a challenge to respondents, since severity can be subjective, even though women's self-report will be the primary option for capturing maternal morbidity in places where women do not usually use skilled care providers or facilities for maternal services. The fact that women who died from obstetric complications were excluded from this study might have some effect on this finding.

\section{Competing interests}

The authors declare that they have no competing interests.

\section{Authors' contributions}

SL: Developed design, participated in the statistical analysis, developed the sequence alignment and drafted the manuscript. ET: Performed the statistical analysis, Participated in the design of the study, participated in the draft of manuscript, Participated in the sequence alignment. TG: Participated in the statistical analysis, helped to develop design and revised manuscript draft. All authors read and approved the final manuscript.

\section{Authors' information}

Serawit Lakew: Lecturer (MSc), Department of Nursing and Midwifery, Arba Minch College of Health Sciences, Arba Minch, South West Ethiopia. Erdaw Tachbele: Lecturer (MSc, PhD cand.), Department of Nursing and Midwifery, Addis Ababa University, Addis Ababa, Ethiopia.

Terefe Gelibo: Lecturer (MPH, PhD student), Department of Public Health, Addis Ababa Science and Technology University, Addis Ababa, Ethiopia.

\section{Acknowledgement}

We would like to forward our gratitude to Addis Ababa University, School of Allied Health Sciences. We thank also Arba Minch Zuria district

administrators, the supervisors, respondents and Data Collectors.

\section{Author details}

'Department of Nursing and Midwifery, Arba Minch College of Health Sciences, Arba Minch, South West Ethiopia. ${ }^{2}$ Department of Nursing and Midwifery, Addis Ababa University, Addis Ababa, Ethiopia. ${ }^{3}$ Department of Public Health, Addis Ababa Science and Technology University, Addis Ababa, Ethiopia.

Received: 29 March 2015 Accepted: 19 November 2015

Published online: 28 November 2015

\section{References}

1. United Nations (UN). The Millennium Development Goals Report 2012. New York: United Nations (UN); 2012.

2. Central Statistical Agency [Ethiopia] and ICF International. Ethiopia Demographic and Health Survey 2011. Addis Ababa, Ethiopia and Calverton, Maryland: Central Statistical Agency and ICF International; 2012. p. 141-56.

3. WHO, Department of Reproductive Health and Research Family and Community Health. Managing Complications in Pregnancy and Child Birth, a guide for Midwifes and Doctors, Geneva. 2003.

4. Centers for Disease Control and Prevention (CDC). CDC 24/7: Saving Lives: Protecting People. CDC - Pregnancy Complications - Reproductive Health [database on the Internet]. 2013. 
5. Federal Democratic Republic of Ethiopia Ministry of Health $(\mathrm{MOH})$ report of 2010. Management Protocol on Selected Obstetrics topics; 2010. p. 1-128.

6. Michael K, Tulshi D, Peter K, and Yasmin A. National Institute of Population Research and Training (NIPORT) report; Bangladesh Maternal Health Services and Maternal Mortality Survey of 2001. Dhaka, Bangladesh and Calverton, Maryland: NIPORT, ORC Macro, Johns Hopkins University, and ICDDR; 2003. p. $75-82$.

7. More NS, Alcock G, Das S, Bapat U, Joshi W, Osrin D. Spoilt for choice? Care-seeking for health problems during pregnancy in Mumbai slums. US National Library of Medicine, National Institutes of Health. 2011;6(7):746-59. doi:10.1080/17441692.2010.520725.

8. Schellenberg J. Maternal and Newborn Health Care, London School of Hygiene and Tropical Medicine, Baseline finding from Ethiopia. 2013. p. 455.

9. Worku AG, Yalew AW, Afework MF. Maternal Complications and Women's Behavior in Seeking Care from Skilled Providers in North Gondar, Ethiopia. PLoS One. 2013:8(3):e60171. doi:10.1371/journal.pone.0060171.

10. Henry OJ, Lagoro KD, Orach CG. Prevalence of Malaria and Treatment Seeking Behaviours among Pregnant Women in Postconflict Internally Displaced Persons' Camps in Gulu District. Hidawi ISRN J. 2012;164935:5. http://dx.doi.org/10.5402/2012/164935.

11. Sharma SK, Vong-Ek P. Perceptions and Care Seeking Behavior of Obstetric Complication in Thailand. Kathmandu Univ Med J. 2012;38(2):63-70.

12. Michael A, Kanta J, Peter K, Tulshi S, Ahmed A, Shams E, et al. Materna Health and Care-Seeking Behavior In Bangladesh: Findings from a National Survey. Int Fam Plan Perspect. 2007:33(2):75-82

13. Anderson FW, Naik SI, Feresu SA, Gebrian B, Karki M, Harlow SD. Perceptions of pregnancy complications in Haiti. Int J Gynecol Obstet. 2008;100:116-23.

14. Okutu D. Access to and utilization of antenatal care services in Uganda. Regional Institute for population Studies, University of Ghana. 2006. p. 1-34.

15. Chakraborty N, Islam A, Chowdhury R, Bari W, Akhter H. Determinants of the use of maternal health services in rural Bangladesh. Health Promotion International. 2003;18(4):327-37. ๑ Oxford University Press.

16. Chiang C, Labeeb SA, Higuchi M, Mohamed AG, Aoyama A. Barriers to the Use of Basic Health Services Among Women in Rural Southern Egypt. Nagoya J Med Sci. 2013;75:225-31.

17. Census. Southern Peoples, Nations and Nationalities Region, Tables 2.1, and 3.4, Ethiopia. 2007. [Online access: Arba Minch Zuria - Wikipedia, the free encyclopedia.htm], retrieved at 12 Jan 2014.

18. SNNPR, Gamo Gofa Zone, Arba Minch Zuria Woreda Health Office Report, 2013.

19. UNFPA State of the World's Midwifery 2011, part 4 country profile report of Ethiopia; 2011. p. 72-73. (www.stateoftheworldsmidwifery.com), retrieved at 30 march 2014.

20. Nigussie M, Mariam DH, Mitike G. Assessment of safe delivery service utilization among women of childbearing age in North Gondar Zone, North West Ethiopia. Ethiop J Health Develop. 2004;18:145-52.

21. Ro"o"st M, Altamirano VC, Liljestrand J, Esse'n B. Does antenatal care facilitate utilization of emergency obstetric care? A case-referent study of earmiss morbidity in Bolivia. Acta Obstetricia Et Gynecologica Scandinavica. 2010;89:335-42.

22. Olasupo P, Liasu A. Perception and Health Seeking behaviour of Nigerian women about pregnancy related risks. J Chin Clin Med. 2007;2(11):643-654.

\section{Submit your next manuscript to BioMed Central and we will help you at every step:}

- We accept pre-submission inquiries

- Our selector tool helps you to find the most relevant journal

- We provide round the clock customer support

- Convenient online submission

- Thorough peer review

- Inclusion in PubMed and all major indexing services

- Maximum visibility for your research

Submit your manuscript at www biomedcentral com/submit 